\title{
The rs1527483, but not rs3212018, CD36 polymorphism associates with linoleic acid detection and obesity in Czech young adults
}

\author{
Jiř́i Plesník ${ }^{1,2}$, Omar Šerý ${ }^{1,3}$, Amira S. Khan ${ }^{2,4}$, Peter Bielik ${ }^{1}$ and Naim A. Khan ${ }^{2 *}$ \\ ${ }^{1}$ Laboratory of Neurobiology and Molecular Psychiatry, Department of Biochemistry, Faculty of Science, Masaryk University, \\ 61137 Brno, Czech Republic \\ ${ }^{2}$ Physiologie de la Nutrition E Toxicologie, UMR U1231 INSERM/Université de Bourgogne-Franche Comté (UBFC)/Agro-Sup, \\ 21000 Dijon, France \\ ${ }^{3}$ Institute of Animal Physiology and Genetics, Academy of Sciences of the Czech Republic, Veverí 97, 60200 Brno, \\ Czech Republic \\ ${ }^{4}$ Laborartoire de Biologie Cellulaire E Moléculaire, Université des Frères Mentouri, 25000 Constantine, Algeria
}

(Submitted 28 June 2017 - Final revision received 10 November 2017 - Accepted 27 November 2017 - First published online 15 February 2018)

\section{Abstract}

Recent evidence has raised the possibility of the existence of a sixth taste modality - that is, taste for fat - which is mediated by lingual CD36 and plays a role in obesity. Consequently, the genetic polymorphism of CD36 has been shown to be associated with altered oro-sensory detection of dietary lipids. In the present study, we investigated the relationship between oro-sensory perception of linoleic acid (LA), two CD36 polymorphisms (rs1527483 and rs3212018), obesity parameters and craving habits for dietary lipids in young Czech adults. We also sequenced 5 and 6 exons of $C D 36$ to trace out any new mutation that might be responsible for the difference in taste perception. We observed that craving for dietary lipids was correlated with anthropometric parameters $(P<0.05)$ and LA detection threshold $(P=0.033)$. The participants with the CC genotype of the rs1527483 polymorphism had lower BMI $(P=0 \cdot 011)$, waist circumference $(P=0 \cdot 005)$, waist:height ratio $(P=0.010)$ and higher sensitivity for LA $(P=0.037)$ than the participants with the CT and TT genotypes. Interestingly, we did not observe any association between the rs3212018 polymorphism and the studied parameters. Moreover, we did not observe any mutation in exons 5 and 6 of the $C D 36$ gene in these subjects. Finally, we can state that rs1527483, but not rs3212018, is associated with high body weight in young Czech subjects.

Key words: CD36: Fat taste: Genetic polymorphism: rs1527483

It has been now well established that there are five basic taste modalities: sweet, sour, bitter, salty and umami. Nevertheless, a growing number of studies have stressed the existence of a sixth taste modality - that is taste of fat $^{(1)}$. Dietary lipids represent an important source of energy. However, if consumed in large quantity, dietary fat may lead to different health complications such as obesity, the metabolic syndrome, cancer, type 2 diabetes mellitus (T2DM) ${ }^{(2)}$ and Alzheimer's disease ${ }^{(3,4)}$. As far as the incidence of obesity is concerned, the Czech Republic ranks at the fourth place in Europe ${ }^{(5)}$.

The oro-sensory perception of dietary lipids is principally mediated by two receptors, GPR120 and CD36, in humans ${ }^{(6,7)}$. CD36 exhibits high affinity for long-chain fatty acids (LCFA), whereas GPR120 is a low-affinity receptor ${ }^{(8)}$. Consequently, genetic variations in $C D 36$ have been shown to influence oral lipid perception. The first study that was focused on the implication of $C D 36 \mathrm{SNP}$ in oral fat sensitivity showed that human participants with the AA genotype of the rs1761667 polymorphism exhibited a higher detection threshold for LCFA compared with subjects with the GG genotype ${ }^{(9)}$. Since this pioneer work, a number of studies have reported similar results

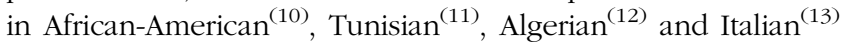
populations.

Besides the rs1761667 SNP, other CD36 SNP such as rs1527483 and rs3212018 are also interesting by the point of view of obesity. Bokor et al. ${ }^{(14)}$ have reported the relationship between the rs1527483 polymorphism (25444 C>T) and obesity, high $\mathrm{BMI}$ and percentage of body fat (BF\%). This SNP is also implicated in high NEFA plasma levels in Caucasians subjects ${ }^{(15)}$. Another study conducted on African-American subjects showed, for the first time, a possible role of the rs1527483 polymorphism in dietary lipids oral perception ${ }^{(10)}$, although a later study did not confirm this finding ${ }^{(13)}$. As regards the rs3212018 polymorphism, Keller et al. have reported that this CD36 polymorphism was

Abbreviations: LA, linoleic acid; WC, waist circumference; WHR, waist:hip ratio; WHtR, waist:height ratio.

* Corresponding author: Professor N. A. Khan, fax +33 3803963 30, email Naim.Khan@u-bourgogne.fr 
associated with BMI and waist circumference (WC) in African-American participants. Moreover, Gautam et al. ${ }^{(16)}$ studied the association of rs3212018 with T2DM in an Indian population.

The primary aim of our study was to determine whether two common CD36 polymorphisms, rs1527483 and rs3212018, play a role in the modulation of oral fatty acid perception and adiposity in young Czech adult participants. Second, we studied the relationship between food preferences, CD36 genotype and anthropometric parameters. Finally, we performed DNA sequencing of exons 5 and 6 encoding hydrophobic pocket of CD $36^{(17)}$ in order to find out any additional polymorphisms/ mutations related to oral sensitivity of dietary lipids.

\section{Methods}

\section{Participants}

In all, 116 young adult Caucasian participants (forty-three male and seventy-three female, age 21.8 (sEm 0.28) years) were recruited in this study. To avoid any influence of age on taste perception $^{(18)}$, the subjects belonged to the same age group. We recruited lean and obese subjects. The participants did not follow any energy or dietary restriction regimen (such as veganism or gluten-free diet). Other exclusion criteria were as follows: significant weight loss in the past 3 months, any medication known to affect appetite, sudden taste loss, regular smoking (more than one packet per week), pregnancy and lactation and medical conditions such as hypertension or diabetes. Before the recruitment for the study, the participants were informed about the aim of the study and the procedures they would undergo. Each person approved and signed a written informed consent. The study was approved by the Ethical Committee of the Masaryk University in Brno.

\section{Anthropometrics}

The parameters were measured without shoes and in light clothing. Body weight was measured with an electronic weight scale with an accuracy of $100 \mathrm{~g}$. Height was measured using a measuring tape with an accuracy of $0.1 \mathrm{~cm}$. Waist and hip circumferences were measured in standing position on the narrowest diameter between xiphoid process and the iliac crest and at the widest part over the greater trochanters, respectively. The circumferences were measured to an accuracy of $0.1 \mathrm{~cm}$. From obtained measurements, we determined the following parameters: $\mathrm{BMI}\left(\mathrm{BMI}=\right.$ weight $/$ height $\left.^{2}, \mathrm{~kg} / \mathrm{m}^{2}\right)$, waist:hip ratio (WHR $=\mathrm{WC} /$ hips circumference, $\mathrm{cm} / \mathrm{cm}$ ) and waist:height ratio $(\mathrm{WHtR}=\mathrm{WC} /$ height, $\mathrm{cm} / \mathrm{cm})$.

\section{Questionnaires}

To observe the craving for dietary lipids, a modification of validated self-administered questionnaire Food Craving Inventory (FCI $)^{(19)}$ was used. The FCI is a questionnaire composed of thirty-seven items and is designed to measure the frequency of cravings for specific food. The food items were divided into four groups: high fats, sweets, carbohydrates/starches and fastfood fats. The participants then rated, from scale 1 'never' to 5 'always/almost every day', how often they craved for each item during the last month of the study. In our study, we were focused only on dietary lipids, and thus we used only the food items that were categorised as 'high fat' and 'fast-food fat'. The 'fatty' food items are very similar in both American and Czech diets, and thus we removed only one item from the 'high-fat' group - that is 'maize bread', which is very uncommon in the Czech diet. Because we used only the lipid-rich food items, we did not perform the same computation as in the original paper. Hence, we calculated the mean craving for each food group and also for both the groups together.

The participants were also asked whether they consumed alcoholic beverages and what was their average consumption per week. On the basis of their answers, we calculated the quantity of consumed alcohol as alcohol units per week.

\section{Oral linoleic acid detection thresholds}

Linoleic acid (LA) detection thresholds were determined according to alternative forced choice $\operatorname{method}^{(20)}$, using ascending concentration of LA (i.e. 0.018, 0.18, 0.37, 0.75, 1.5, 3, 6 and $12 \mathrm{mmol} / \mathrm{l})$ in deionised water containing Arabic gum $(5 \%, \mathrm{w} / \mathrm{v})$ and EDTA $(0 \cdot 01 \%, \mathrm{w} / \mathrm{v})$. Control samples contained only Arabic gum (5\%, w/v) and EDTA $(0 \cdot 01 \%, \mathrm{w} / \mathrm{v})$. All chemicals were purchased from Sigma-Aldrich. The emulsion was homogenised using sonication probe MS 72 (Bandelin). The samples were diluted and immediately used or stored at $4^{\circ} \mathrm{C}$ in the absence of light. To prevent oxidation, the samples were stored in oxygen-free atmosphere (pure $\mathrm{N}_{2}$ ). The samples were used within $48 \mathrm{~h}$.

The day before the tasting session, the participants were instructed to avoid smoking and consumption of alcoholic beverages and hot spicy meals (such as chilli, garlic or horseradish). On the tasting day, the participants were suggested to come early in the morning without taking breakfast (fasting state).

During the tasting session, the participants were subjected to a set of three solutions. Each set contained two control samples and one sample with LA. The subjects were instructed that in each set there is only one odd sample, and they were unknown to the nature of the tested molecule (such as oily or bitter). The participants kept each sample in their mouths for a few seconds. They were not allowed to drink the solution; rather, they had to spit it out. The participants were asked to rinse their mouth with deionised water between each concentration used. To mask visual and olfactory cues, participants wore a nose clip and a blindfold. After each set, the participants were asked to identify the odd samples containing LA. When an incorrect response was given, we increased the LA concentration. The procedure was terminated when the subject correctly identified the solution containing the LA three times.

\section{CD36 polymorphisms genotyping}

Genomic DNA was isolated from buccal swabs using DNA extraction system Prepito (PerkinElmer) based on paramagnetic particles separation. The isolated DNA was used to determine genotypes of two CD36 gene polymorphisms: rs1527483, which encodes C/T substitution, and rs3212018, which encodes 16-bp deletion. We used restriction fragment length polymorphism 
method using Taq thermostable DNA polymerase (Kapa Biosystems). To genotype rs1527483, a 235-bp fragment was amplified using forward 5'-GCCAATTAGAATCACTTCATAAACC-3' and reverse 5'-TGATGGATTAAACCCAAATGAA-3' primers. PCR primers for rs3212018 were as follows: forward 5'-TCTGGGA GAAATGAGATAAAAGATG-3' and reverse 5'-GCAGCAA TCCTGGTCTTATG-3', with a 182-bp PCR product. Both polymorphisms were amplified by the same protocol using annealing temperature at $56^{\circ} \mathrm{C}$.

For the rs1527483 polymorphism, a 236-bp product was digested by TaqI restriction enzyme (Thermo Fisher Scientific). The fragments were as follows: 107 and 129 bp (T-allele) and 37, 70 and 129 bp (C-allele). Regarding the rs3212018 polymorphism, the PCR product had one fragment of $182 \mathrm{bp}$ in case of wild type (insertion), and in case of deletion one 166-bp product was observed. The PCR products from the both polymorphisms were separated by $2 \%(\mathrm{w} / \mathrm{v})$ agarose gel, stained with ethidium bromide.

\section{CD36 sequencing}

We sequenced exons 5 and 6, which encode hydrophobic pocket, a part of extracellular domain of CD36 that is responsible for fatty acid binding ${ }^{(17)}$. These exons encode amino acids $95-143$ (exon 5) and 144-203 (exon 6). Out of our study group, we selected subjects who were absolutely unable (no correct response in the taste test) to detect LA ( $n$ 4) and the same number of subjects with lowest detection threshold for LA (supertaster). First, we amplified selected regions using standard PCR. Sequences of used primers and temperatures profile are displayed in the Table 1 . The $5^{\prime}$ end of the forward primers started $31 \mathrm{bp}$ and $122 \mathrm{bp}$ before exon 5 and 6, respectively, and the $5^{\prime}$ end of the reverse primers started 110 and $170 \mathrm{bp}$ behind exons 5 and 6, respectively. The PCR product was then purified using Shrimp Alkaline Phosphatase and Exonuclease I (Thermo Fisher Scientific). The mixtures were incubated at $37^{\circ} \mathrm{C}$ for $15 \mathrm{~min}$ and $85^{\circ} \mathrm{C}$ for $15 \mathrm{~min}$ to inactivate the enzymes, followed by sequencing with BigDye ${ }^{\circledR}$ Terminator version 3.1 (Thermo Fisher Scientific) with the forward PCR primers as sequencing primers. The amplicons were then purified using EDTA/ethanol precipitation, resuspended in $10 \mu \mathrm{l}$ of Hi-Di Formamide (Thermo Fisher Scientific) and sequenced on an automated ABI 3130 Genetic Analyzer (Thermo Fisher Scientific). The resulting sequences were analysed in MEGA 7 software $^{(21)}$ and NC_000007.14 sequence from Nucleotide NCBI database was used for alignment.

\section{Data analyses}

Statistical analyses were conducted using Statistica 12 software (StatSoft) and the data are presented as means with their standard errors. According to the Shapiro-Wilk test, most parameters were found to be non-normally distributed, and thus non-parametric tests were used in each test. To compare two variables, the Mann-Whitney $U$ test was used; in the case of three variables, Kruskal-Wallis ANOVA was used. For correlation between various parameters, Spearman's rank correlation was performed. The two-tailed Fisher's exact test was used for comparison of allelic frequencies. Hardy-Weinberg equilibrium was tested by the $\chi^{2}$ test. $P$ values $\leq 0.05$ were considered statistically significant.

\section{Results}

\section{Subjects descriptive statistics}

On the basis of BMI, we observed twelve obese participants with $\mathrm{BMI}>30 \mathrm{~kg} / \mathrm{m}^{2}$ in our study. Out of the twelve obese subjects, six were male and six were female. Table 2 shows the anthropometric parameters. We noticed higher BMI, WHR, WHtR and WC in the male group compared with the female group. As expected, we observed a strong positive association for all four measured parameters $(R>0.32, P<0.01)$. The average age of the participants was $21 \cdot 84$ (SEM $0 \cdot 22$ ) years.

\section{Questionnaires}

We noticed a positive relationship between all anthropometric parameters and craving for 'high-fat' food items. The participants who tend to have more frequent craving for fatty food had larger BMI, WC, WHR and WHtR ( $P$ values, respectively, $0.026,0.007,0.011$ and 0.044). In addition, LA detection threshold was high in subjects who often craved for 'high-fat' food items $(P=0.033)$. The 'fast-food fat' items did not, however, associate with any one of the parameters. Interestingly, the consumption of alcohol is associated with 'fast-food fat' items. The subjects who consumed more alcohol exhibited more frequent craving for 'fast-food fat' food items $(P=0 \cdot 021)$. The obese participants reported more frequent craving for lipids items than controls; nonetheless, the difference was statistically marginal $(P=0 \cdot 05)$. The list of food items and exact $P$ values are displayed in Table 3.

\section{Linoleic acid sensitivity}

Out of 116 subjects, eleven subjects were classified as non-tasters who failed to detect LA even at the highest concentration of the fatty acid used in the study. We observed a positive correlation between LA detection threshold and all the parameters - for example, BMI, WC, WHR and WHtR (Fig. 1, P values 0.047, 0.004,

Table 1. Sequences of the primers and the temperature profile used for sequencing of $C D 36$ exons 5 and 6

\begin{tabular}{|c|c|c|c|c|c|c|c|c|}
\hline Exon & Primers & & & Temper & profile & & & Product length (bp) \\
\hline \multirow[t]{2}{*}{5} & 5'-AAATGTTTTTGAATTTTGTTTACTGCT-3' & \multirow{3}{*}{$\begin{array}{l}94^{\circ} \mathrm{C} \\
3 \min \end{array}$} & \multicolumn{3}{|c|}{$40 x$} & \multirow[b]{2}{*}{$72^{\circ} \mathrm{C}$} & \multirow[b]{2}{*}{$10^{\circ} \mathrm{C}$} & \multirow[t]{2}{*}{296} \\
\hline & 5'-CGTTTTGATAAAAATGGAAAAACA-3' & & $94^{\circ} \mathrm{C}$ & $54^{\circ} \mathrm{C}$ & $72^{\circ} \mathrm{C}$ & & & \\
\hline 6 & $\begin{array}{l}\text { 5'-TGGCAGGATCTGGCAGTAA-3' } \\
\text { 5'-TTCCCAACTAGGAAAGCTGAA-3' }\end{array}$ & & $30 \mathrm{~s}$ & $60 s$ & $30 \mathrm{~s}$ & $5 \min$ & $\infty$ & 492 \\
\hline
\end{tabular}


Table 2. Characteristics of the study group* (Mean values with their standard errors)

\begin{tabular}{|c|c|c|c|c|c|c|c|}
\hline \multirow[b]{2}{*}{ Parameters } & \multicolumn{2}{|c|}{ Total $(n 116)$} & \multicolumn{2}{|c|}{ Boys ( $n$ 43) } & \multicolumn{2}{|c|}{ Girls ( $n 73)$} & \multirow[b]{2}{*}{$P$} \\
\hline & Mean & SEM & Mean & SEM & Mean & SEM & \\
\hline Age (years) & 21.84 & 0.22 & 21.91 & 0.37 & $21 \cdot 80$ & 0.28 & NS \\
\hline BMI $\left(\mathrm{kg} / \mathrm{m}^{2}\right)$ & 23.34 & 0.42 & 24.61 & 0.63 & 22.53 & 0.53 & 0.003 \\
\hline WC $(\mathrm{cm})$ & 76.88 & 1.01 & 84.00 & 1.69 & $72 \cdot 87$ & 0.94 & $>0.001$ \\
\hline WHR $(\mathrm{cm} / \mathrm{cm})$ & 0.79 & 0.01 & 0.83 & 0.01 & 0.77 & 0.01 & $>0.001$ \\
\hline WHtR $(\mathrm{cm} / \mathrm{cm})$ & 0.44 & 0.00 & 0.46 & 0.01 & 0.43 & 0.01 & 0.005 \\
\hline LA thresholds $(\mathrm{mmol} / \mathrm{l})$ & 1.47 & 0.27 & 2.51 & 0.59 & 0.88 & 0.23 & 0.003 \\
\hline
\end{tabular}

WC, waist circumference; WHR, waist:hip ratio; WHtR, waist:height ratio.

* The $P$ values were obtained by using the Mann-Whitney $U$ test

Table 3. Correlations between Food Craving Inventory ( $\mathrm{FCl}$ ) food item groups and measured adiposity parameters, linoleic acid detection threshold and weekly alcohol consumption in the participants $(n 116)^{*}$

\begin{tabular}{|c|c|c|c|c|c|c|}
\hline \multirow[b]{3}{*}{ Parameters } & \multicolumn{6}{|c|}{ Food item group } \\
\hline & \multicolumn{2}{|c|}{ Fast-food fats } & \multicolumn{2}{|c|}{ High fats } & \multicolumn{2}{|c|}{ Total fats } \\
\hline & $R$ & $P$ & $R$ & $P$ & $R$ & $P$ \\
\hline BMI $\left(\mathrm{kg} / \mathrm{m}^{2}\right)$ & 0.163 & NS & 0.225 & 0.026 & 0.211 & 0.037 \\
\hline$W C(\mathrm{~cm})$ & $0 \cdot 151$ & NS & 0.277 & 0.007 & 0.238 & 0.022 \\
\hline WHR $(\mathrm{cm} / \mathrm{cm})$ & 0.162 & NS & 0.263 & 0.011 & 0.239 & 0.021 \\
\hline WHtR (cm/cm) & $0 \cdot 161$ & NS & 0.212 & 0.044 & 0.201 & 0.056 \\
\hline LA thresholds $(\mathrm{mmol} / \mathrm{l})$ & 0.173 & NS & 0.214 & 0.033 & 0.187 & 0.062 \\
\hline WAC (unit/week) & 0.224 & 0.021 & 0.182 & 0.060 & 0.210 & 0.030 \\
\hline
\end{tabular}

(a)

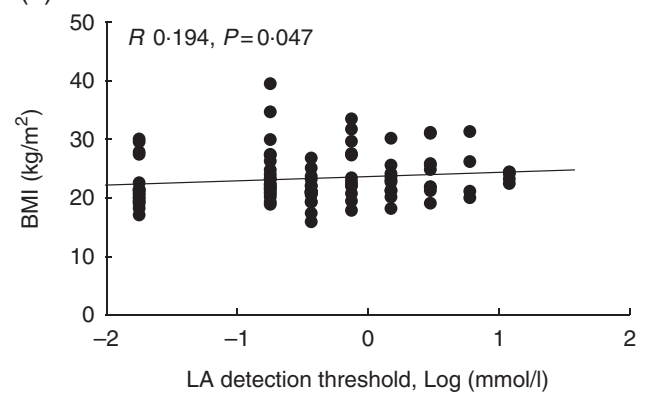

(c)

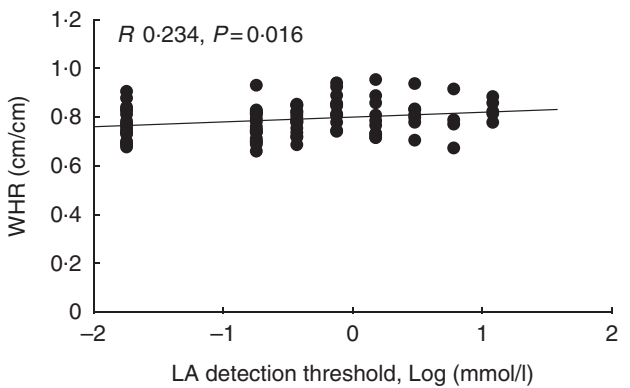

(b)

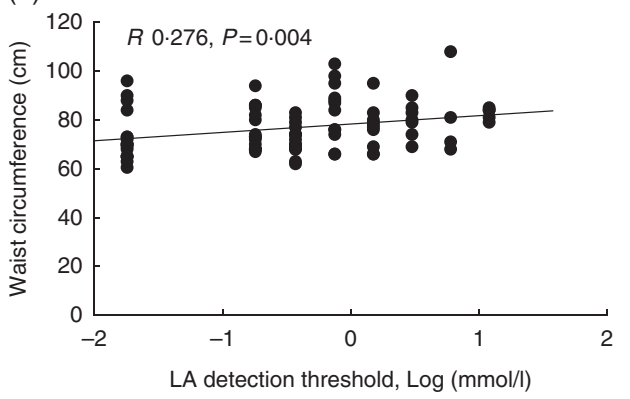

(d)

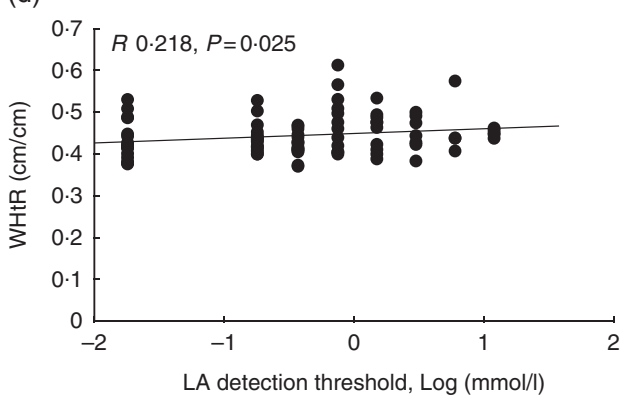

Fig. 1. Correlation between adiposity parameters and linoleic acid (LA) detection threshold ( $n$ 116). We observed a positive correlation between LA acid detection threshold and BMI (a), waist circumference (b), waist:hip ratio (WHR) (c) and waist:height ratio (WHtR) (d). The $R$ and $P$ values were obtained using Spearman's rank correlation. 
0.016, 0.025, respectively). The male subjects had almost 3-foldhigher detection threshold (lower sensitivity) than the female participants $(P=0.003)$. We did not observe any difference in the detection threshold between obese and lean participants. However, LA oral detection threshold correlated with the craving for 'high-fat' food items $(P=0.033)$ but not with 'fast-food fat' items according to the FCI questionnaire (Table 3 ).

\section{Genetic analyses}

The studied polymorphisms did not deviate from HardyWeinberg equilibrium. We obtained $\chi^{2}$ values of $1.40(P=0.24)$ and $0.00(P=1)$ for $\mathrm{rs} 1527483$ and $\mathrm{rs} 3212018$, respectively. To maximise the number of subjects in each group, we combined into one group the subjects with minor homozygous genotypes and those with heterozygous genotypes (e.g. TT + CC for rs1527483 and DD + ID for rs3212018). The genotype and allelic frequencies are shown in Table 4 . The CT/TT genotypes $(P=0.007)$ and T-allele $(P=0.004)$ were more frequent in the obese group. The participants with CT/TT genotypes had higher BMI, WC and WHtR than the participants with the CC genotype ( $P$ values were $0.011,0.005$ and 0.010 , respectively). Nonetheless, the difference in WHR was only marginally nonsignificant $(P=0.077)$ (Fig. 2). We also noticed that subjects

Table 4. Genotype and allelic frequencies of $C D 36$ polymorphisms (Numbers and percentages)

\begin{tabular}{lccrrrrr}
\hline Polymorphisms & \multirow{2}{*}{ Variation } & Genotype & $n$ & $\%$ & Allele & $n$ & $\%$ \\
\hline rs1527483 & \multirow{2}{*}{ C/T } & CC & 97 & 83.6 & C & 211 & 90.9 \\
& & CT & 17 & 14.7 & T & 21 & 9.1 \\
rs3212018 & \multirow{2}{*}{16 bp del } & TT & 2 & 1.7 & & & \\
& & ins/ins & 77 & 66.4 & ins & 189 & 81.5 \\
& & ins/del & 35 & 30.2 & del & 43 & 18.5 \\
& & del/del & 4 & 3.5 & & & \\
\hline
\end{tabular}

(a)

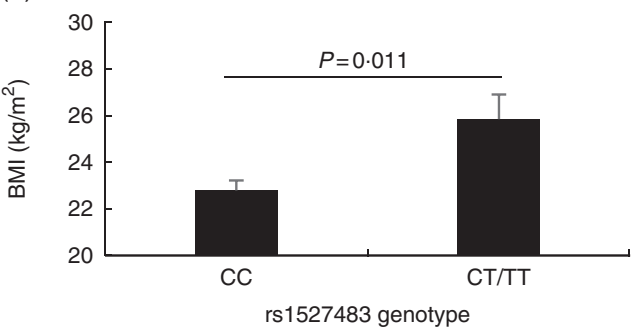

(c)

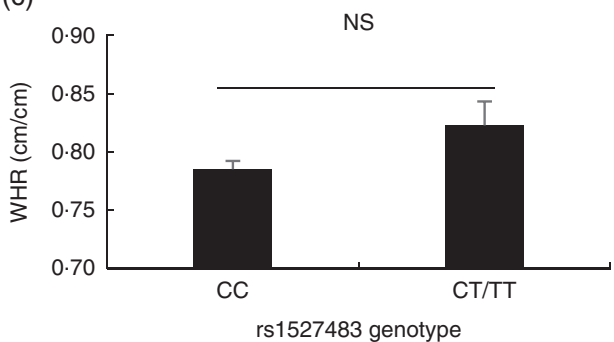

with CT/TT genotypes were less sensitive to LA (higher threshold) than participants with the CC genotype $(P=0.037$, Fig. 3). We did not observe any significant association between rs3212018 and the measured parameters.

The sequenced exons 5 and 6 corresponded to sequences, described on an online database. We sequenced base pairs $80661027-80661322$ (exon 5) and base pairs $80662855-$ 80663346 (exon 6) on chromosome 7. In the analysed DNA sequences of four non-tasters and supertasters, we did not find any polymorphism.

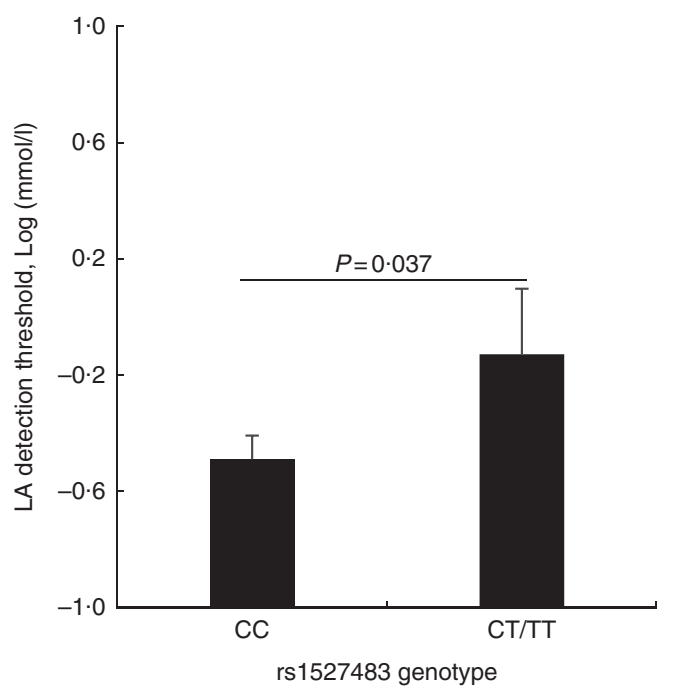

Fig. 3. Relationship between the CD36 rs1527483 genotype and linoleic acid (LA) detection threshold. Subject with CT or TT genotypes ( $n$ 19) had significantly higher LA detection threshold than subjects with the CC genotype ( $n$ 97). $P$ values were obtained using the Mann-Whitney $U$ test. Values are means, with their standard errors represented by vertical bars.

(b)

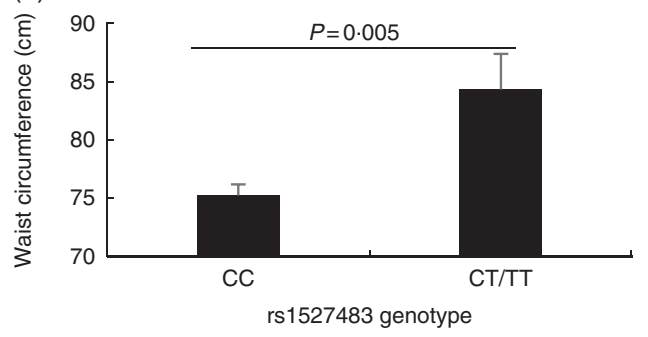

(d)

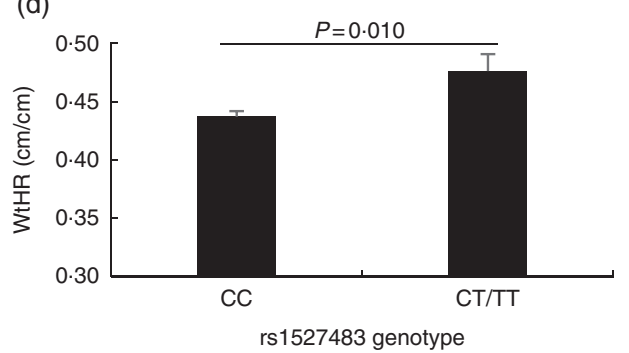

Fig. 2. Association between measured adiposity parameters and the CD36 rs 1527483 genotype. The participants with CT or TT genotypes ( $n$ 19) had significantly larger BMI (a), waist circumference (b) and waist:height ratio (WHtR) (d), but not WHR (c), than subjects with the CC genotype ( $n$ 97). $P$ values were obtained using the Mann-Whitney $U$ test. Values are means, with their standard errors represented by vertical bars. 


\section{Discussion}

Dietary perception involves a combination of several senses - that is, vision, olfaction, somatosensation and taste ${ }^{(22)}$. It has been recently shown that dietary lipids are perceived by olfactory and somatosensory systems ${ }^{(23)}$. Moreover, a gustatory cue might also be involved in the perception of dietary lipids ${ }^{(1)}$.

It is generally accepted that dietary habits and food preferences play an important role in the development of adiposity. We observed that craving for 'fat' food was associated with adiposity parameters. Some of the food items in the FCI questionnaire are rich in saturated and trans-unsaturated fatty acids, and overconsumption of these lipids has been previously associated with high $\mathrm{BMI}$, obesity and $\mathrm{T} 2 \mathrm{DM}^{(24-26)}$. We noticed that increased craving for these food items was associated with high oral detection threshold for LA. This observation agrees well with previous reports, demonstrating that intake of dietary lipids might result in decreased oral lipid sensitivity in humans ${ }^{(27,28)}$ and rodents ${ }^{(8)}$.

Our study also shows that obese subjects exhibited high detection thresholds for LA; however, the values were not statistically significantly different owing to the small number of participants (data not shown). Nonetheless, there was a positive correlation between LA detection threshold and measured adiposity parameters (i.e. BMI, WC, WHR and WHtR). Our previous studies conducted on Algerian children ${ }^{(12)}$ and teenagers ${ }^{(28)}$ also showed a significant positive correlation between oleic acid detection threshold and WC and BMI, respectively. BMI is currently the most used method to appreciate adiposity in the populations worldwide. However, if the study group is composed of subjects with high lean/muscle mass, the BMI values may be misinterpreted $^{(29)}$. To eliminate this misinterpretation, we additionally calculated other adiposity parameters. All the parameters were in strong positive correlation. Interestingly, we also noticed a significantly high detection threshold in male participants. Similarly, Ong et $a l^{(30)}$ showed that female subjects exhibited higher oral lipid sensitivity than male participants.

Our major findings are related to rs1527483 and LA oral detection. Hence, participants with the CC genotype were more sensitive to LA detection than subjects with CT/TT genotypes. This is the first study that shows a statistically significant relationship between this SNP and dietary lipid oral detection threshold in Czech subjects. Our observations corroborate to a few studies that have established a plausible association between rs1527483 with oral fat perception of lipids in African-American and Asian participants ${ }^{(10,30)}$, although a study conducted on Italian population did not confirm these findings ${ }^{(13)}$. The discrepancy between these results might be owing to two major aspects: the study of Keller et al. included relatively high number of subjects (more than 300 participants) ${ }^{(10)}$, whereas the study of Melis et al. was performed only on sixty-four subjects (with only seven subjects with the CT genotype and none with the TT genotype $)^{(13)}$. The difference might also arise from the techniques used to assess fatty acid detection sensitivity. In fact, Melis et al. used a paper disc, impregnated with oleic acid, placed on the centre of the tongue, which means no contact with circumvallate papillae, located on the posterior region of the tongue, whereas the tasting method used by our group ${ }^{(31)}$ and Keller et al. involved gargling with LA, which means that the fatty acid can reach the whole tasting area. We do not know the expression level of CD36 in different taste papillae in humans, although CD36 expression in circumvallate papillae and foliate papillae is, respectively, 9-fold and 3.5-fold higher than that in fungiform papillae in mice ${ }^{(7)}$.

In the past, the T-allele of rs1527483 was also associated with high $\mathrm{BMI}$ and $\mathrm{BF} \%{ }^{(14)}$. Despite the fact that we did not measure the $\mathrm{BF} \%$ in our study, we used several adiposity parameters, and as expected we observed the same trend. Moreover, we observed a higher T-allele frequency in our obese group than the lean subjects, but, as mentioned earlier, the obese group was significantly smaller than the control group; hence, this observation should be confirmed in the future. In another study, no association between this SNP and BMI in Polish children was found ${ }^{(32)}$. However, this study was conducted on children with type 1 diabetes, and thus we cannot compare this study with our results. The rs3212018 polymorphism was previously associated with obesity and $\mathrm{WC}^{(10)}$, but we failed to confirm these findings. Moreover, a large meta-analysis of Choquet et al. ${ }^{(33)}$ failed to observe any differences between CD $36 \mathrm{SNP}$ and BMI or obesity. To observe a clear role of rs3212018, a larger study group is required in the future.

To date, no clear explanation on how rs1527483 might influence CD36 function is available. In the past, Love-Gregory et al. $^{(34)}$ showed that some of $C D 36 \mathrm{SNP}$ may reduce $C D 36$ expression in platelets and monocytes. This study also included rs1527483, but no difference was observed for this polymorphism. The study by Chevrot et al. also showed that decreased expression of $C D 36$ directly influences oral sensitivity for fatty acids in rodents ${ }^{(35)}$. CD36 is known to be expressed in a number of cells, including endothelial cells, adipocytes, erythroblasts or striated muscle cells ${ }^{(36)}$. Thus, it may be possible that the rs1527483 polymorphism influences CD36 function in other tissues. The rs1527483 polymorphism is located in intron 11 of CD36, and intronic SNP may have a regulatory role as published elsewhere $^{(37)}$. However, the exact physiological consequence of the rs1527483 polymorphism is still unclear. We tried to observe whether the craving for lipid-rich food items is related to CD36 genotypes, but we failed to observe any significant differences.

It is, thus, possible that a mutation located in the CD36 pocket may cause the dietary lipids oral insensitivity. In our study, eleven subjects were classified as LA non-tasters. We sequenced exons 5 and 6, which encode the region surrounding lysine-164. We did not find any mutation in this area, neither in selected non-tasters nor in supertasters. Thus, if there is a mutation that may determine LCFA oral insensitivity, it may be located outside of the hydrophobic pocket of CD36, or in the intronic region. This particular phenomenon requires further studies in future.

In conclusion, our study brings further evidence that oral perception of dietary lipids is influenced by CD36 polymorphisms. Moreover, the rs1527483 genotype and LA oral detection threshold are associated with adiposity parameters.

\section{Acknowledgements}

This work has been supported by Agency for Healthcare Research, Czech Republic (AZV CR) - grant project no. NV16-29900A.

One of the authors (J. P.) is thankful to the French Embassy in Prague for the sanction of the scholarship that enabled him to stay in Dijon for his PhD. 
N. A. K. designed the study. J. P. conducted the research (data collection and SNP analysis). P. B. conducted DNA sequencing. J. P. performed the statistical analysis. O. S. supervised the hands-on conduct of the experiment and provided the facilities for the data collection. J. P., O. S. and N. A. K. wrote the manuscript. A. S. K participated in data analysis and linguistic correction of the manuscript. All authors have read and approved the final content of the manuscript.

The authors declare that there are no conflicts of interest.

\section{References}

1. Besnard P, Passilly-degrace P \& Khan NA (2016) Taste of fat: a sixth taste modality? Physiol Rev 96, 151-176.

2. Garduno-Diaz SD \& Khokhar S (2012) Prevalence, risk factors and complications associated with type 2 diabetes in migrant South Asians. Diabetes Metab Res Rev 28, 6-24.

3. Hanson AJ, Bayer-Carter JL, Green PS, et al. (2013) Effect of apolipoprotein $\mathrm{E}$ genotype and diet on apolipoprotein $\mathrm{E}$ lipidation and amyloid peptides. JAMA Neurol 70, 972-980.

4. Šerý O, Povová J, Míšek I, et al. (2013) Molecular mechanisms of neuropathological changes in Alzheimer's disease: a review. Folia Neuropathol 51, 1-9.

5. Spilková J (2016) Teenage overweight and obesity: a pilot study of obesogenic and obesoprotective environments in the Czech Republic. Morav Geogr Reports 24, 55-64.

6. Ichimura A, Hirasawa A, Poulain-Godefroy O, et al. (2012) Dysfunction of lipid sensor GPR120 leads to obesity in both mouse and human. Nature 483, 350-354.

7. Laugerette F, Passilly-Degrace P, Patris B, et al. (2005) CD36 involvement in orosensory detection of dietary lipids, spontaneous fat preference, and digestive secretions. J Clin Invest 115, 3177-3184.

8. Ozdener MH, Subramaniam S, Sundaresan S, et al. (2015) CD36- and GPR120-mediated $\mathrm{Ca}^{2+}$ signaling in human taste bud cells mediates differential responses to fatty acids and is altered in obese mice. Gastroenterology 146, 995-1005.

9. Pepino MY, Love-Gregory L, Klein S, et al. (2012) The fatty acid translocase gene CD36 and lingual lipase influence oral sensitivity to fat in obese subjects. J Lipid Res 53, 561-566.

10. Keller KL, Liang LC, Sakimura J, et al. (2012) Common variants in the CD36 gene are associated with oral fat perception, fat preferences, and obesity in African Americans. Obesity (Silver Spring) 20, 1066-1073.

11. Mrizak I, Šerý O, Plesnik J, et al. (2015) The A allele of cluster of differentiation 36 (CD36) SNP 1761667 associates with decreased lipid taste perception in obese Tunisian women. $\mathrm{Br}$ J Nutr 113, 1330-1337.

12. Sayed A, Šerý O, Plesnik J, et al. (2015) AA genotype is associated with decreased lipid taste perception in young obese, but not lean, children. Int J Obes 39, 920-924.

13. Melis M, Sollai G, Muroni P, et al. (2015) Associations between orosensory perception of oleic acid, the common single nucleotide polymorphisms (rs1761667 and rs1527483) in the CD36 gene, and 6-n-propylthiouracil (PROP) tasting. Nutrients 20, 2068-2084.

14. Bokor S, Legry V, Meirhaeghe A, et al. (2010) Singlenucleotide polymorphism of CD36 locus and obesity in European adolescents. Obesity 18, 1398-1403.

15. Ma X, Bacci S, Mlynarski W, et al. (2004) A common haplotype at the CD36 locus is associated with high free fatty acid levels and increased cardiovascular risk in Caucasians. Hum Mol Genet 13, 2197-2205.

16. Gautam S, Agrawal CG \& Banerjee M (2015) CD36 gene variants in early prediction. Genet Test Mol Biomarkers 19, 144-149.
17. Kuda O, Pietka TA, Demianova Z, et al. (2013) Sulfo$\mathrm{N}$-succinimidyl oleate (SSO) inhibits fatty acid uptake and signaling for intracellular calcium via binding CD36 lysine 164. J Biol Chem 288, 15547-15555.

18. Mojet J, Christ-Hazelhof E \& Heidema J (2001) Taste perception with age: generic or specific losses in threshold sensitivity to the five basic tastes? Chem Senses 26, 845-860.

19. White MA, Whisenhunt BL, Williamson DA, et al. (2002) Development and validation of the food-craving inventory. Obes Res 10, 107-114.

20. Chalé-Rush A, Burgess JR \& Mattes RD. (2007) Multiple routes of chemosensitivity to free fatty acids in humans. Am J Physiol Gastrointest Liver Physiol 292, 1206-1212.

21. Kumar S, Stecher G \& Tamura K (2016) MEGA7: molecular evolutionary genetics analysis version 7.0 for bigger datasets brief communication. Mol Biol Evol 33, 1870-1874.

22. Rolls ET (2012) Taste, olfactory and food texture reward processing in the brain and the control of appetite. Proc Nutr Soc 71, 488-501.

23. Heinze JM, Preissl H, Fritsche A, et al. (2015) Physiology \& behavior controversies in fat perception. Physiol Behav 152, 479-493.

24. Phillips CM, Kesse-Guyot E, McManus R, et al. (2012) High dietary saturated fat intake accentuates obesity risk associated with the fat mass and obesity-associated gene in adults. J Nutr 142, 824-831.

25. Schulze MB, Manson JE, Willett WC, et al. (2003) Processed meat intake and incidence of type 2 diabetes in younger and middle-aged women. Diabetologia 46, 1465-1473.

26. Wang Y \& Beydoun MA (2010) Meat consumption is associated with obesity and central obesity among US adults. Int J Obes 33, 621-628.

27. Stewart JE \& Keast RSJ (2012) Recent fat intake modulates fat taste sensitivity in lean and overweight subjects. Int J Obes $\mathbf{3 6}$, 834-842.

28. Daoudi H, Plesník J, Sayed A, et al. (2015) Oral fat sensing and CD36 gene polymorphism in Algerian lean and obese teenagers. Nutrients $\mathbf{4}$, 9096-9104.

29. Garrido-Chamorro R, Sirvent-Belando J, Gonzalez-Lorenzo M, et al. (2009) Correlation between body mass index and body composition in elite athletes. J Sport Med Phys Fit 49, 278-284.

30. Ong HH, Tan YN \& Say YH (2017) Fatty acid translocase gene CD36 rs1527483 variant influences oral fat perception in Malaysian subjects. Physiol Behav 168, 128-137.

31. Karmous I, Plesník J, Khan AS, et al. (2017) Orosensory detection of bitter in fat-taster healthy and obese participants: genetic polymorphism of CD36 and TAS2R38. Clin Nutr (Epublication ahead of print version 21 June 2017).

32. Luczyński W, Fendler W, Ramatowska A, et al. (2014) Polymorphism of the FTO gene influences body weight in children with type 1 diabetes without severe obesity. Int J Endocrinol 2014, 10-14.

33. Choquet H, Labrune Y, De Graeve F, et al. (2011) Lack of association of CD36 SNPs with early onset obesity: a meta-analysis in 9,973 European subjects. Obesity (Silver Spring) 19, 833-839.

34. Love-Gregory L, Sherva R, Schappe T, et al. (2011) Common CD36 SNPs reduce protein expression and may contribute to a protective atherogenic profile. Hum Mol Genet 20, 193-201.

35. Chevrot M, Bernard A, Ancel D, et al. (2013) Obesity alters the gustatory perception of lipids in the mouse: plausible involvement of lingual CD36. J Lipid Res 54, 2485-2494.

36. Silverstein R \& Febbraio M (2010) CD36, a scavenger receptor involved in immunity, metabolism, angiogenesis, and behavior. Sci Signal 2, 1-16.

37. Millar DS, Horan M, Chuzhanova NA, et al. (2010) Characterisation of a functional intronic polymorphism in the human growth hormone (GH1) gene. Hum Genomics 4, 289-301. 